Editorial

\title{
Whither the impending european regulation of presumed endocrine disruptors?
}

The legislative impulse to regulate presumed endocrine disrupting chemicals (EDCs) was born as an appendage to the US Food Quality Protection Act of 1996, focusing on public health rather than environmental issues. Pressed by advocacy claims, US legislators were persuaded by a study in animals - shortly after officially labeled as scientific misconduct ${ }^{1}$ - and by epidemiologic claims that could not be validated. Twenty years later, the momentum to regulate EDCs has spread worldwide, even though many studies over the last decades have yet to yield credible epidemiologic evidence of public health adversities linked to xenoendocrine contaminants. Absent a confirmed public health target, what could justify a program to regulate presumed EDCs?

The European Commission in June 2016 issued draft criteria for EDCs identification and regulation, still set in the conjectural frameworks of regulatory science and precautionary considerations. ${ }^{2}$ Animal and reductionist laboratory tests are to be used because tests in humans are not possible, and arbitrary definitions of adverse effects are to be adopted as valid clinical proxies for humans. As a novel challenge, the drafted criteria disregard potency in identifying EDC hazards, contrary to plain empirical evidence, common sense and elementary thermodynamics. Clearly, without sufficient causal potencies nothing stirs in the natural universe, including endocrine-dependent events.

A group of experts hosted by the German Federal Institute for Risk Assessment (BfR), included potency in the identification of EDC hazards and proposed that any substance could be considered an EDC, if acting by an endocrine mode of action (MoA) and causing adverse effects in a daily dose range up to1000 $\mathrm{mg} / \mathrm{Kg} \mathrm{bw}{ }^{3}$ The equivalent upper dose would be $70 \mathrm{~g}$ daily for a $70 \mathrm{Kg}$ person. On these grounds, and invoking precaution, regulators likely would prescribe the highest doses as the standard for EDC testing, parallel to the prescribed maximum tolerated dose (MTD) for carcinogen bioassays. Such a testing regimen would likely indict numerous

\footnotetext{
1 Office of Research Integrity, US National Institutes of Health, Bethesda, Maryland USA. Available at: http://grants.nih.gov/grants/guide/notice-files/NOT-OD-02003.html.

${ }^{2}$ Commission of the European Union. Setting out scientific criteria for the determination of endocrine disrupting properties and amending Annex II to Regulation (EC) 1107/2009. Ref: Ares (2016)3071834-29/06/2016. PART-2016-154327V1.pdf; PART-2016-154328V1.pdf.

3 Scientific principles for the identification of endocrine disrupting chemicals - a consensus statement. The German Federal Institute for Risk Assessment, Berlin, Germany. April 11-12, 2016. http://www.bfr.bund.de/en/international_expert_ meeting_on_endocrine_disruptors-197246.html.
}

substances, even though typical xenoendocrines show receptor binding affinities thousands of times lower than human physiologic hormones, and are commonly experienced at very low concentrations. A regulatory scheme on these terms would considerably reduce vegetal food supplies by banning a large segment of staple foods that carry low levels of phytoestrogens. It would also ban many cosmetics, medicines and other compounds containing low levels of natural and synthetic hormones.

With these prospects, and conceding that regulation of putative EDCs may not be resisted, the adoption of pertinent World Health Organization (WHO/IPCS) testing guidelines has been considered. ${ }^{4}$ Excluding reductionist laboratory assays, the guidelines endorse tests in appropriate whole animal models to reach estimates of potencies and NOAELs against human hormone standards. On this evidence, it would be questionable to estimate human risks in the absence of clinical epidemiologic benchmarks, but it would be sensible to exonerate and remove from public anxieties those substances testing positive below appropriate thresholds of toxicologic or regulatory concern (TTC/TRC), at realistic exposures. Substances exceeding those thresholds would be regulated, although few of such instances could likely be found, due to the absence of clinical epidemiologic signals and the low concentrations and receptor binding affinities of putative xenoendocrines.

Opposing such options, a European EDC regulatory program disregarding potency would reinforce a dangerous precedent by further encouraging the creative regulation of putative hazards for putative public health adversities. The Commission did ask for public comments on the drafted criteria, but the first question is why the Commission chose to embark on this course. Is the Commission intrigued by the prospect of a new open season of authoritarian regulations justified by the flimsiest conjectures? Does the Commission hope for a flood of protests to counteract advocacy pressures, and thus to reinstate potency as a core justification of EDCs regulation?

Potency or no potency, however, EDC regulation in Europe - and similarly worldwide - is posed to continue in an autocratic rather than factual mode: it will be set by the same administrators writing rules, policing, prosecuting, judging, and penalizing. They also will appoint juries of advisors, selected by conflict of interest criteria

\footnotetext{
${ }^{4}$ WHO/IPCS. 2016. Environmental Health Criteria 240. Principles and Methods for the Risk Assessment of Chemicals in Food. Chapter 4 Hazard Identification and Characterization: Toxicological and Human Studies. http://apps.who.int/iris/ bitstream/10665/44065/7/WHO_EHC_240_7_eng_Chapter4.pdf?ua=1.
} 
designed to preclude dissent. The arbitrary extent of such proceedings is generally unknown to the public and to most elected legislators, happy to believe that regulations are grounded on testable evidence. If sufficiently informed of this whimsical, authoritarian and legally enforced reality, free societies would be hard pressed to tolerate regulations that cause massive economic misallocations and pervasive public anxieties. More so if the public realized that even putative health and longevity benefits of EDC regulation could not be assessed, in the absence of testable clinical and epidemiologic benchmarks of public health adversities linkable to EDCs.

The social, civic and moral implications of such regulatory scenarios should advise restraint while raising some key questions. Remembering how the raison d'être of regulation is to provide public health benefits, is it reasonable to issue costly regulations for the relief of putative public health problems bereft of clinical epidemiologic footprints? If a case is made for preventive and precautionary regulations, should those be justified by direct or indirect evidence that is factually measurable, or should they be triggered by whimsical conjectures? More pointedly, are certain regulations becoming a pretext for lucrative debates about angels on pin heads? What special interests stand to benefit from regulation, other than public health at large? Have biases and conflicts of interest of all participants - regulators included - been made transparent and openly perceived by the public?

Ethical and rational answers to these questions could put regulation on the right course, but it will not be. What inflames controversial regulations at the center of public, administrative and legislative attention is not a yearning for public health or a respect for science. Rather, it is the achieving of compromises to balance the open and ulterior interests of advocacies, academicians, regulators, advisors, industry, politicians and the media. Public health hardly emerges in those discussions.

Under irresistible pressure from advocates, the market, the media and an ill-informed European parliament, the European Commission is proposing to regulate EDCs based on criteria that cannot be justified scientifically or by common sense. They would enable an arbitrary approach to precaution, unconcerned that precaution itself becomes a very costly if not a paralyzing hazard, when its potency and dimensions were not accounted for. Indeed we seem to have reached a decision point: either we return to embrace the evidentiary ethic of science and a sensible approach to socially affordable precaution, or we proceed to slouch toward an "everything goes" regulatory morass dominated by opportunistic special interests. For the moment, and unless improbable legislative miracles may happen, the balance rests in the hands of the European Commission.

Herman N. Autrup, PhD, Emeritus Professor Institute of Public Health, University of Aarhus, Aarhus, Denmark E-mail address: ha@ph.au.dk.

Sir Colin L. Berry, MD, Professor Emeritus of Pathology Queen Mary, London, UK

E-mail address: colin@sircolinberry.co.uk.

Samuel M. Cohen, MD, PhD, Professor Department of Pathology and Microbiology, University of Nebraska Medical Center, Omaha, NE, USA E-mail address: scohen@unmc.edu.
E. Edmond Creppy, PhD, Professor of Toxicology Department of Toxicology and Applied Hygiene, University of Bordeaux, Bordeaux, France E-mail address: eecreppyprof@yahoo.fr.

João L. de Camargo, MD, PhD, Professor of Pathology UNESP Medical School, Botucatu 18600 SP, Brazil E-mail address: decam@fmb.unesp.br.

Wolfgang Dekant, PhD, Professor of Toxicology University of Wuerzburg, Wuerzburg, Germany

E-mail address: dekant@toxi.uni-wuerzburg.de.

Daniel Dietrich, PhD, Professor Human and Environmental Toxicology, University of Konstanz, Konstanz, Germany

E-mail address: Daniel.Dietrich@uni-konstanz.de.

Corrado L. Galli, PhD, Professor

Section of Toxicology and Risk Assessment, Department of Pharmacological and Biomolecular Sciences, University of Milan, Milan, Italy

E-mail address: corrado.galli@unimi.it.

Jay I. Goodman, PhD, Professor of Pharmacology and Toxicology Michigan State University, East Lansing, MI, USA E-mail address: goodman3@msu.edu.

Gio B. Gori, MPH, DSc, Editor-in-Chief Regulatory Toxicology and Pharmacology, Bethesda, MD, USA

Helmut A. Greim, MD, Emeritus Professor Toxicology, Technical University of Munich, Munich, Germany E-mail address: helmut.greim@1rz.tum.de.

James E. Klaunig, PhD, Professor Department of Environmental Health, Indiana University, Bloomington, IN 47405, USA E-mail address: jklauni@indiana.edu.

Marcello Lotti, MD, Professor Department of Cardiology, Thoracic and Vascular Sciences, School of Medicine, University of Padua, Padua, Italy E-mail address: marcello.lotti@unipd.it.

Hans W. Marquardt, MD, Professor Emeritus Experimental and Clinical Toxicology, University of Hamburg Medical School. Hamburg, Germany

E-mail address: marquardt@uke.uni-hamburg.de.

Kendall B. Wallace, PhD, Professor University of Minnesota Medical School, Duluth, MN, USA E-mail address: kwallace@d.umn.edu.

Hiroshi Yamazaki, PhD, Professor of Drug Metabolism and Pharmacokinetics

Showa Pharmaceutical University, Machida, Tokyo, Japan E-mail address: hyamazak@ac.shoyaku.ac.jp.

${ }^{*}$ Corresponding author.

Available online 9 September 2016 